\title{
Oculomotor nerve palsy as a preceding symptom of adult sporadic Burkitt lymphoma: A case report and review of the literature
}

\author{
YUN LIANG, LUYIN DING, XIAN LI, WEIQIN WANG and XIAOHONG ZHANG \\ Department of Hematology, The Second Affiliated Hospital of Zhejiang University School of Medicine, \\ Hangzhou, Zhejiang 310009, P.R. China
}

Received October 16, 2015; Accepted August 19, 2016

DOI: $10.3892 / 01.2017 .5583$

\begin{abstract}
Adult sporadic Burkitt lymphoma (BL) is a rare aggressive neoplasm and represents $\sim 1-5 \%$ of all lymphomas diagnosed in adults. The disease exhibits an aggressive clinical manifestation, which frequently involves the central nervous system (CNS) in the early stages, and is usually accompanied by abnormalities in cerebrospinal fluid (CSF) examinations and/or neuroradiography. The current study describes the rare case of a 29-year-old man who presented with oculomotor nerve palsy without palpated masses, B symptoms, peripheral blood anomaly and abnormalities of the nervous system. The patient was initially misdiagnosed with abducens diplopia, but was subsequently confirmed to have BL with early CNS invasion. Epstein-Barr virus and human immunodeficiency virus infection were negative. Intensive systemic chemotherapy with hyperfractionated cyclophosphamide, vincristine, therarubicin and dexamethasone (hyper-CVAD, cyclophosphamide $600 \mathrm{mg} / \mathrm{m}^{2}$ on days $2-4$; vincristine $1.4 \mathrm{mg} / \mathrm{m}^{2}$ on days 5 and 12 , therarubicin $50 \mathrm{mg} / \mathrm{m}^{2}$ on day 5 and dexamethasone $40 \mathrm{mg}$ on days 2-5 and 12-15), including intrathecal chemotherapy with methotrexate $(5 \mathrm{mg})$, arabinocytidine $(5 \mathrm{mg})$ and dexamethasone (5 mg), was determined as an appropriate treatment. Rituximab (375 mg/m $\mathrm{m}^{2}$ on day 1) was administered alongside chemotherapy to increase treatment efficacy. The patient temporarily underwent complete remission, but subsequently relapsed as no suitable bone marrow donor was available. Adult sporadic BL with early CNS invasion, which traditional tests such as neuroradiography and CSF examination fail to identify, is uncommon. Therefore, appropriate diagnostic tests are critical for accurate diagnosis and must be performed immediately, particularly in patients that present with unusual image and laboratory manifestations.
\end{abstract}

Correspondence to: Professor Xiaohong Zhang, Department of Hematology, The Second Affiliated Hospital of Zhejiang University School of Medicine, 88 Jiefang Road, Hangzhou, Zhejiang 310009, P.R. China

E-mail: debby1021@163.com

Key words: oculomotor nerve palsy, Burkitt lymphoma, rituximab

\section{Introduction}

Burkitt lymphoma (BL) is a type of B cell non-Hodgkin lymphoma (NHL), which most commonly presents in children and young adults (1). Three subtypes of BL have been defined: i) The endemic form occurring primarily in Africa, which usually affects children and adolescents, and is associated with Epstein-Barr virus (EBV) infection; ii) the adult sporadic form; and iii) the immunodeficiency-associated form, occurring primarily in patients infected with the human immunodeficiency virus (HIV) $(1,2)$.

$\mathrm{BL}$ is a clinically aggressive tumor with bulky observable masses, B symptoms and frequent involvement of extranodal sites (2). Bone marrow infiltration and leptomeningeal central nervous system (CNS) involvement are also common at the time of diagnosis (3). The association between $\mathrm{BL}$ and mature B cell acute lymphoblastic leukemia (ALL) has previously been recognized, and these two nomenclatures may represent different manifestations of a single disease rather than two separate diseases $(3,4)$.

BL tumor cells are characterized by the expression of B cell-specific surface markers, including immunoglobulin (Ig) M, cluster of differentiation (CD) 19 and CD20, and by Burkitt-like morphology according to the French-American-British classification (4). The chromosomal translocation $\mathrm{t}(8 ; 14)$, which results in the juxtaposition of the IgH region on chromosome 14 with the MYC gene from chromosome 8 , has been identified in nearly all cases of BL $(5,6)$. BL is prone to invade the CNS during the early stages of the disease, and this invasion is usually accompanied by abnormalities in cerebrospinal fluid (CSF) examinations and/or neuroradiography $(7,8)$.

The present study describes the symptoms and treatment regime of an adult patient with BL and CNS invasion, who presented with a sudden onset of right oculomotor nerve palsy without abnormal neuroradiography, CSF examinations and initial peripheral blood.

\section{Case report}

A 29-year-old man was admitted to the Department of Hematology, The Second Affiliated Hospital of Zhejiang University School of Medicine (Hangzhou, China) on 6 November, 2013 with a 1-week history of a drooping right eyelid and impaired 
eye-movement. The patient also suffered from double vision without apparent etiology, but did not experience symptoms of fever, chills, headache, numbness, vomiting, glare, halos, starbursts, sweating, weight loss or trauma, and had no history of smoking, alcohol or drug abuse. No significant abnormalities were reported in the family medical history.

Physical examination revealed that the pupils were round and symmetrical, with a diameter of $3.0 \mathrm{~mm}$, and the patient had normal vision, visual field and fundus, in addition to direct and indirect light reflex. The right eye exhibited impaired downside and abduction movements; however, normal closure action and other neural functions, including facial sensation, hearing, pronunciation and swallowing, were normal. The patient was able to walk and move freely without numbness of limbs. No enlargement of lymph nodes, liver or spleen was observed.

Laboratory tests revealed a normal blood count on day 1 , which was as follows: White blood cell (WBC) count, 5.8x10 $/ 1$ (normal range, 4.0-10.0x10 $/ 1$ ); hemoglobin (Hb) count, $133 \mathrm{~g} / 1$ (normal range, 110-160 g/l); platelet count, 150x10 $/ 1$ (normal range, 100-300x10 $/ 1$ ); neutrophilic granulocyte percentage, $62.1 \%$ (normal range, 50.0-70.0\%); lymphocyte percentage, $27.4 \%$ (normal range, 20.0-40.0\%); and monocyte percentage, $9.3 \%$ (normal range, 4.0-12.0\%). Serum lactate dehydrogenase (LDH) and erythrocyte sedimentation rate were $907 \mathrm{U} / 1$ (normal range, 100-248 U/l) and $90 \mathrm{~mm} / \mathrm{h}$ (normal range, 0-15 mm/h), respectively. Serum albumin, $\mathrm{Ig}$, acute phase reactive protein, renal and liver function tests were all within normal limits. Viral serological examinations, including hepatitis $\mathrm{A}, \mathrm{B}$ and $\mathrm{C}$ virus, HIV, EBV and cytomegalovirus were all negative. B-mode ultrasonography exhibited no marked abnormalities in the liver, gallbladder, pancreas or spleen, and no significant enlargement of lymph nodes was detected. Magnetic resonance angiography of the brain with intravenous gadolinium was unremarkable, and an enhanced computed tomography (CT) scan indicated that orbital and optic nerve were normal. Furthermore, cerebrovascular diseases, including thrombosis, infraction and hemorrhagic apoplexy, were excluded by cerebral angiography. Though there were no signs of meningeal irritation, a lumbar puncture was performed and CSF examination was normal: WBC count, 0/ $\mu 1$ (normal range, $0-5 / \mu 1$ ); red blood cell count, 0/ $\mu \mathrm{l}$ (normal range, $0-5 / \mu \mathrm{l}$ ); glucose level, $3.7 \mathrm{mmol} / \mathrm{l}$ (normal range, $2.8-4.5 \mathrm{mmol} / \mathrm{l}$ ); and protein level, $23.30 \mathrm{mg} / \mathrm{dl}$ (normal range, $15-40 \mathrm{mg} / \mathrm{dl}$ ). Neither abnormal cells nor microorganisms were identified in the centrifugal extraction of CSF.

The patient was initially diagnosed with abducens diplopia which was considered to have developed from a non-specific type of single cranial neuritis. The patient was subsequently provided with methylprednisolone ( $5 \mathrm{mg}$, oral tid) and vitamin B12 (0.5 mg, intramuscular injection qd) to take for $\sim 1$ week. However, the condition of the patient did not improve, and arthralgia and bone pain developed in the sacrum and pelvis. A magnetic resonance imaging (MRI) exam was performed, which revealed diffuse abnormal signals in bones, although no marked bone destruction was identified on X-ray or CT images. Re-examination of peripheral blood 8 days later revealed slight decrease in $\mathrm{Hb}$ level to $122 \mathrm{~g} / 1$ (normal range, 110-160 g/l), while WBC and platelet counts remained within normal limits. However, LDH levels had escalated rapidly to 2,452 U/1 (normal range, 100-248 U/1). At day 13, symptoms of anemia became more severe; however, WBC and platelet counts remained normal (Table I). A peripheral blood smear was subsequently performed revealing atypical lymphocytes and immature cells. Bone marrow examination identified extreme hyperplasia with excess blast cells $>59 \%$ (normal range, $<5 \%$ ) and inhibited normal hematopoiesis. Microscopically, tumor cells were characterized by a diffuse monotonous pattern of medium-sized, round nuclei with clumped chromatin, deep basophilic cytoplasm and easily identified cytoplasmic vacuoles, all hallmarks of Burkitt-like cell morphology (Fig. 1). Immunophenotype analysis demonstrated positive expression of membrane antigens, including CD20, CD22, CD19, CD38, CD10, CD22, CD79a and human leukocyte antigen-D related, which supported the diagnosis of mature B lineage ALL with a Ki-67 index $>90 \%$. There was no significant expression of the following markers: CD3, CD5, CD23, CD34, terminal deoxynucleotidyl transferase (TdT), B cell lymphoma-2 (Bcl-2), melanoma associated antigen-1 (MUM-1) or myeloperoxidase. The expression of all available leukemia fusion genes was negative and included breakpoint cluster region/ABL, myeloid/lymphoid or mixed-lineage leukemia (MLL)-AFX, MLL-AF1p; MLL/AF4, dupMLL; MLL/ENL, E2A/pre B-cell leukemia homeobox 1, SIL/T-cell acute lymphocytic leukemia 1, homeobox 11, TEL/acute myeloid leukemia 1 and TEL/Abelson murine leukemia viral oncogene homolog 1 . Cytogenetic studies of 16 metaphases identified 46, XY and 4 chromosome abnormalities as 45, X, -Y, t(8;14)(q24;q32).

A bone marrow biopsy was subsequently performed to check for unusual lumps and tangible swollen lymph nodes. Body macrophages were dispersed among sheets of non-cohesive cells, presenting a typical 'starry sky' morphological appearance. Immunohistochemistry (IHC) was performed using antibodies purchased from Beijing Zhongshan Golden Bridge Biotechnology Co., Ltd., Beijing, China) at a dilution of 1:100. These antibodies were used including CD19 (\#TA506234), CD20 (\#TA800394), CD22 (\#TA807826), CD79a (\#TA800688), CD3 (\#TA320268), CD5 (\#TA501335), CD23 (\#TA801531), TdT (\#TA327989), Bcl-2 (\#ZA-0536), Bcl-6 (\#TA804186), MUM-1 (\#ZA-0583) and cyclin D1 (\#sc-753). The results demonstrated positive expression of CD19, CD20, CD22 and CD79a, and negative expression of CD3, CD5, CD23, TdT, Bcl-2, Bcl-6, MUM-1 and cyclin D1. Therefore, the diagnosis of BL/mature B cell ALL with CNS involvement was confirmed and the patient received combined chemotherapy with hyperfractionated cyclophosphamide, vincristine, therarubicin and dexamethasone, and an additional rituximab (R-hyper-CVAD, rituximab $375 \mathrm{mg} / \mathrm{m}^{2}$ on day 1 ; cyclophosphamide $600 \mathrm{mg} / \mathrm{m}^{2}$ on days $2-4$; vincristine $1.4 \mathrm{mg} / \mathrm{m}^{2}$ on days 5 and 12 ; therarubicin $50 \mathrm{mg} / \mathrm{m}^{2}$ on day 5 and dexamethasone $40 \mathrm{mg}$ on days 2-5 and 12-15). The patient also underwent aggressive intrathecal chemotherapy with methotrexate $(5 \mathrm{mg})$, arabinocytidine $(5 \mathrm{mg})$ and dexamethasone $(5 \mathrm{mg})$ twice a week during intravenous chemotherapy. Following treatment, CSF analysis exhibited a transparent appearance with normal chemistry and cytological manifestation. Complete remission and negative minimal residual disease (MRD) in the bone marrow were achieved following induction chemotherapy, and palsy and double vision of the right eye also improved. The patient was administered 3 further cycles of combined chemotherapy of R-hyper-CVAD and intrathecal chemotherapy. However, no suitable donor could 
Table I. Complete blood counts and LDH of the present patient at the time of initial diagnosis of abducens diplopia, 8 days later during follow-up and at the established diagnosis of BL.

\begin{tabular}{lccc}
\hline BL parameter & $\begin{array}{c}\text { Initial diagnosis of } \\
\text { abducens diplopia }\end{array}$ & Follow-up & At BL diagnosis \\
\hline Time, days & 1 & 8 & 13 \\
Hemoglobin, $\mathrm{g} / \mathrm{l}$ & 133 & 122 & 102 \\
Leukocytes, $\mathrm{x} 10^{\%} / 1$ & 5.8 & 5.4 & 5.4 \\
Lymphocyte, $\%$ & 27.4 & 29.5 & 22.4 \\
Neutrophils, $\%$ & 62.1 & 62.8 & 67.8 \\
Monocytes, $\%$ & 9.3 & 6.9 & 4.0 \\
Platelets, $\mathrm{x} 10^{9} / 1$ & 150 & 149 & 120 \\
Peripheral blood blasts, $\%$ & - & - & 4.0 \\
Serum LDH, U/l & 907 & 2452 & 3896 \\
\hline
\end{tabular}

LDH, lactate dehydrogenase; BL, Burkitt lymphoma.

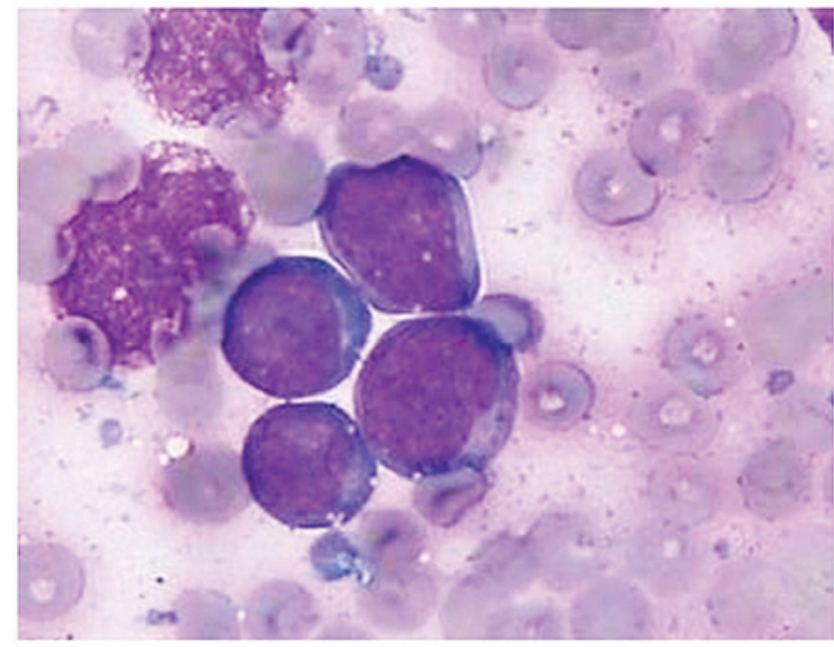

Figure 1. Morphological features of bone marrow smears 13 days after hospital admission (day 13). Wright-Giemsa-stained bone marrow exhibited an excess of Burkitt-like cells (original magnification, $\mathrm{x} 1,000$ ).

be located in time for the patient to undergo the hematopoietic stem cell transplantation required to prevent relapse. The patient experienced relapse with recurrent positive MRD prior to the last cycle of chemotherapy and finally succumbed to BL.

\section{Discussion}

Whether EBV-positive or negative, $>80 \%$ of patients with BL or mature B cell ALL are known to have the $t(8 ; 14)$ (q24;q32) chromosome translocation, which juxtaposes the MYC-encoding gene to an Ig enhancer element (IgEE) $(9,10)$. As IgEEs are specifically active in mature B cells, this translocation to MYC results in aberrantly high MYC expression, which enhances B cell proliferation regardless of whether EBV infection has occurred (10). Other chromosomal aberrations may also induce rapid cell proliferation, which results in a highly aggressive clinical course (11).

Adults with BL frequently present with B symptoms, bulky masses and laboratory evidence of tumor lysis; in addition, CNS involvement occurs in $\sim 70 \%$ of patients diagnosed (4). The pathology of CNS leukemia affects the entire nervous system, including spinal cords, cranial nerves, cerebral hemispheres and associated nerve roots. The diagnosis of CNS leukemia is based on neurological dysfunction, leukemia cells infiltrating the CSF, and/or abnormal neuroradiography on enhanced CT and MRI scans $(12,13)$. However, it is extremely rare that a patient with neurological deficits does not exhibit any abnormalities in either CSF or neuroradiography, and therefore BL is easily neglected or misdiagnosed. Negative CSF cytology may serve as a false negative signal or a possible former stage of the disease.

Previous studies have demonstrated that a number of patients with CNS infiltration exhibited normal CSF cytology initially and required repeated lumbar punctures (14-16). Therefore, multiple lumbar punctures and a flow cytometry analysis of CSF may aid the diagnosis of patients with BL (17). CT and MRI scans have been the principal imaging modalities for the staging and restaging of lymphoma during the last few decades (7); however, they are not accurate at identifying the early symptoms of lymphoma. Therefore, novel neuroimaging methods that may improve early diagnosis of the disease, thus improving patient prognosis, are required. In recent years, a number of studies have demonstrated that information provided by 18 -fluorodeoxyglucose positron-emission tomography/CT (FDG-PET/CT) may result in greater sensitivity compared with anatomic imaging modalities $(18,19)$.

In addition to oculomotor nerve palsy, the elevated level of serum LDH and bone pain experienced by the current patient is suggestive of early abnormal cell proliferation in bone marrow, despite the initial results of the peripheral blood cell count being normal. It is well established that serum LDH levels are an independent prognostic factor in BL, and a higher $\mathrm{LDH}$ level, like that experienced by the present patient, is associated with advanced-stage disease and a less favorable outcome (20). Meanwhile, the abnormal MRI results of the current patient indicated that sacrum and pelvis bone pain was associated with bone marrow dysfunction. Though the peripheral blood WBC and platelet counts were initially within the normal ranges, a gradual change was observed from normal to anemia and, finally, to anemia with blasts. 
The survival rates of patients with BL/mature B cell ALL, particularly for children and adolescents, have improved due to the recent development of aggressive short-term chemotherapy, with a 5-year event-free survival rate of $85-90 \%$ (11). By contrast, the majority of adults and elderly patients cannot tolerate the adverse side effects of aggressive chemotherapy, including drug toxicity and severe infections. Therefore, optimum chemotherapeutic regimens for high-grade BL have not yet been well established $(21,22)$.

Several studies have recently reported high cure rates following the use of similar chemotherapeutic protocols in adults with BL. BL patients who received aggressive short-term chemotherapy demonstrated better overall survival rate than those who received chemotherapy with cyclophosphamide, doxorubicin, vincristine and prednisolone (also known as CHOP), which is routinely administered to patients with non-Hodgkin's lymphoma (23). Prophylactic cranial irradiation and prolonged maintenance have not demonstrated any proven benefits and are not currently recommended for treatment of the disease. Furthermore, introduction of the anti-CD20 monoclonal antibody, rituximab, and/or allogeneic hematopoietic stem transplantation has significantly improved outcomes of adult patients, particularly those that are high-risk $(24,25)$. The poor prognosis of the current patient may have resulted from several adverse prognostic factors, including CNS attacks, complicated karyotype, a high level of $\mathrm{LDH}$ and relapse following first-line treatment.

In conclusion, to the best of our knowledge, the present case is the first to document oculomotor nerve palsy with normal neuroradiography and CSF examination results as a preceding symptom of adult sporadic BL with chromosomal translocation $\mathrm{t}(8 ; 14)$. Such atypical patients present major clinical challenges, including harnessing novel biological technologies to improve risk stratification, defining the value of prognostic factors and developing innovative therapies, particularly for relapse and refractory patients.

\section{References}

1. Turner JJ, Morton LM, Linet MS, Clarke CA, Kadin ME, Vajdic CM, Monnereau A, Maynadié M, Chiu BC, Marcos-Gragera R, et al: InterLymph hierarchical classification of lymphoid neoplasms for epidemiologic research based on the WHO classification (2008): Update and future directions. Blood 116: e90-e98, 2010.

2. Harris NL, Jaffe ES, Diebold J, Flandrin G, Muller-Hermelink HK, Vardiman J, Lister TA and Bloomfield CD: The World Health Organization classification of neoplastic diseases of the hematopoietic and lymphoid tissues. Report of the clinical advisory committee meeting, Airlie house, Virginia, November, 1997. Ann Oncol 10: 1419-1432, 1999.

3. van Besien K, Ha CS, Murphy S, McLaughlin P, Rodriguez A, Amin K, Forman A, Romaguera J, Hagemeister F, Younes A, et al: Risk factors, treatment, and outcome of central nervous system recurrence in adults with intermediate-grade and immunoblastic lymphoma. Blood 91: 1178-1184, 1998.

4. Blum KA, Lozanski G and Byrd JC: Adult Burkitt leukemia and lymphoma. Blood 104: 3009-3020, 2004.

5. Hecht JL and Aster JC: Molecular biology of Burkitt's lymphoma. J Clin Oncol 18: 3707-3721, 2000.

6. ar-Rushdi A, Nishikura K, Erikson J, Watt R, Rovera G and Croce CM: Differential expression of the translocated and the untranslocated c-myc oncogene in Burkitt lymphoma. Science 222: 390-393, 1983.

7. Johnson KA, Tung K, Mead G and Sweetenham J: The imaging of Burkitt's and Burkitt-like lymphoma. Clin Radiol 53: 835-841, 1998.
8. Cairo MS, Gerrard M, Sposto R, Auperin A,Pinkerton CR, Michon J, Weston C, Perkins SL, Raphael M, McCarthy K, et al: Results of a randomized international study of high-risk central nervous system B non-Hodgkin lymphoma and B acute lymphoblastic leukemia in children and adolescents. Blood 109: 2736-2743, 2007.

9. Klein G: Burkitt lymphoma-a stalking horse for cancer research? Semin Cancer Biol 19: 347-350, 2009.

10. Hayday AC, Gillies SD, Saito H, Wood C, Wiman K, Hayward WS and Tonegawa S: Activation of a translocated human c-myc gene by an enhancer in the immunoglobulin heavy-chain locus. Nature 307: 334-340, 1984.

11. Miles RR, Arnold S and Cairo MS: Risk factors and treatment of childhood and adolescent Burkitt lymphoma/leukaemia. Br J Haematol 156: 730-743, 2012.

12. Pui CH and Thiel E: Central nervous system disease in hematologic malignancies: Historical perspective and practical applications. Semin Oncol 36 (4 Suppl 2): S2-S16, 2009.

13. Jang SJ, Yoon DH, Kim S, Yoon S, Kim DY, Park CS, Huh J, Lee SW,Lee DH, Rvu JS and Suh C: A unique pattern of extranodal involvement in Korean adults with sporadic Burkitt lymphoma: A single center experience. Ann Hematol 91: 1917-1922, 2012.

14. Crivii SM, Neamtu S and Bocsan G: Neuroimmunitary profile estimation in cerebrospinal fluid and its importance in childhood acute lymphoblastic leukaemia. Arch Geschwulstforsch 59: 199-204, 1989.

15. Yadav $\mathrm{J}$ and Jain V: Cushing syndrome related to leukemic infiltration of the central nervous system. J Pediatr Endocrinol Metab 28: 717-719, 2015.

16. Bromberg JE, Breems DA, Kraan J, Bikker G, van der Holt B, Smitt PS, van den Bent MJ, van't Veer M and Gratama JW: CSF flow cytometry greatly improves diagnostic accuracy in CNS hematologic malignancies. Neurology 68: 1674-1679, 2007.

17. Quijano S, López A, Manuel Sancho J, Panizo C, Debén G, Castilla C, Antonio García-Vela J, Salar A, Alonso-Vence N, González-Barca E, et al: Identification of leptomeningeal disease in aggressive B-cell non-Hodgkin's lymphoma: Improved sensitivity of flow cytometry. J Clin Oncol 27: 1462-1469, 2009.

18. Carrillo-Cruz E, Marín-Oyaga VA, Solé Rodríguez M, Borrego-Dorado I, de la Cruz Vicente F, Quiroga Cantero E, Manzanares Pérez M, Capote FJ, Ramírez Sánchez MJ, Espigado Tocino I, et al: Role of 18F-FDG-PET/CT in the management of Burkitt lymphoma. Eur J Haematol 94: 23-30, 2015.

19. Just PA, Fieschi C, Baillet G, Galicier L, Oksenhendler E and Moretti JL: 18F-fluorodeoxyglucose positron emission tomography/computed tomography in AIDS-related Burkitt lymphoma. AIDS Patient Care STDS 22: 695-700, 2008.

20. Mintzer DM, Andreeff M, Filippa DA, Jhanwar SC, Chaganti RS and Koziner B: Progression of nodular poorly differentiated lymphocytic lymphoma to Burkitt's-like lymphoma. Blood 64: 415-421, 1984.

21. Mead GM, Barrans SL, Qian W, Walewski J, Radford JA, Wolf M, Clawson SM, Stenning SP, Yule CL, Jack AS, et al: A prospective clinicopathological study of dose modified CODOX-M/IVAC in patients with sporadic Burkitt lymphoma defined using cytogenetic and immunophenotypic criteria (MRC/NCRI LY10 trial). Blood 112: 2248-2260, 2008.

22. Smeland S, Blystad AK, Kvaløy SO, Ikonomou IM, Delabie J, Kvalheim G, Hammerstrøm J, Lauritzsen GF and Holte H: Treatment of Burkitt's/Burkitt-like lymphoma in adolescents and adults: A 20-year experience from the Norwegian Radium Hospital with the use of three successive regimens. Ann Oncol 15: 1072-1078, 2004.

23. Todeschini G, Bonifacio M, Tecchio C, Balter R, Carli G, Stefani PM, Adami F, Zamò A, Dei Tos AP, Marino F, et al: Intensive short-term chemotherapy regimen induces high remission rate (over 90\%) and event-free survival both in children and adult patients with advanced sporadic Burkitt lymphoma/leukemia. Am J Hematol 87: 22-25, 2012.

24. Thomas DA, Faderl S, O'Brien S, Bueso-Ramos C, Cortes J, Garcia-Manero G, Giles FJ, Verstovsek S, Wierda WG, Pierce SA, et al: Chemoimmunotherapy with hyper-CVAD plus rituximab for the treatment of adult Burkitt and Burkitt-type lymphoma or acute lymphoblastic leukemia. Cancer 106: 1569-1580, 2006.

25. Barnes JA, Lacasce AS, Feng Y, Toomey CE, Neuberg D, Michaelson JS, Hochberg EP and Abramson JS: Evaluation of the addition of rituximab to CODOX-M/IVAC for Burkitt's lymphoma: A retrospective analysis. Ann Oncol 22: 1859-1864, 2011. 